\title{
Central European Cooperative Oncology Group
}

With the aim to coordinate clinical trials in oncology according to the standards of Good Clinical Practice in the area of Centraland South-Eastern Europe, the foundation of CECOG was laid in 1999. CECOG defined its mode of interaction based upon respect and mutual esteem in its Atlanta Declaration in 2000. Shortly thereafter, CECOG established both a scientific as well as an advisory board necessary for its functioning and embarked upon two phase III clinical trials in metastatic breast cancer (MBC) and in non-small cell lung cancer (NSCLC), respectively. Both of these studies were published.

In the meantime, CECOG has evolved into a true international cooperative study group being impressively active in the region of Central- and South-Eastern Europe and beyond. CECOG cooperates with more than 150 academic centers in a total of 23 countries, including Austria, Bosnia, Bulgaria, Croatia, Cyprus, Czech Republic, Estonia, Greece, Hungary, Israel, Latvia, Lebanon, Lithuania, Montenegro, Poland, Portugal, Romania, Russia, Serbia, Slovenia, Slovakia, South Africa, and Turkey.

CECOG has not only been formed and supported by many outstanding individuals from the geographic area, but has also proved to be a quality-oriented body with clearly defined decision lines acknowledged and certified by both governmental and industry-driven audits. During the past years, CECOG terminated 15 clinical trials in which almost 2,700 patients have been enrolled. CECOG's clinical trials include the fields of metastatic breast cancer, non-small cell lung cancer, metastatic colorectal cancer, gastroesophageal cancer, gastrointestinal stromal tumors (GIST) and various aspects of both maintenance and dose-dense treatment of malignant disorders. All finalized studies have been published in top or standard journals either in full length or in abstract form.

Besides the purely scientific dimension, all our studies are aimed at enhancing the quality of life for cancer patients by identifying the most suitable and effective treatment under various conditions. A new and ambitious project of CECOG is the initiative 'CECOG 2020'. It is the aim of this initiative to focus on personalized medicine which is the challenge of the future. This project comprises a series of possibilities for cooperation as CECOG can provide a total solution for biopharmaceutical companies seeking rapid drug development and approval by offering strategic development planning, scientific advice and immediate trial execution and evaluation necessary to answer important questions in the area of oncology, based on promising animal and early phase I data translated into consequent clinical testing. CECOG has demonstrated to have the capability, expertise, strategic partnerships to focus and drive to deliver accurate and reliable results within the agreed period of each clinical study. Due to the close cooperation with fast recruiting centers and an impeccable track record, CECOG can deal with new clinical or scientific challenges very quickly.

CECOG's range of activity is, however, not only limited to the conduct of clinical trials but also concentrates on education and the definition of quality standards in regard to the care of patients with malignant diseases. This is done by gathering oncologists from all over the world in order to establish internationally accepted consensus on minimal standards of diagnosis and medical treatment of non-small cell lung cancer, metastatic breast cancer and metastatic colon cancer. The results of CECOG's consensus conferences have been published in leading journals. Moreover, CECOG is proud to be the responsible organizer of the Skeletal Care Initiative. With this approach in education we believe to have made a contribution to an amelioration in medical care of patients not only in our geographic area, which has impressively gained importance, but also in the international oncological community in other parts of the world.

\section{KARGER}

Fax +497614520714

Information@Karger.de

www.karger.com (c) 2010 S. Karger GmbH, Freiburg 